\title{
Implementation of Spread-Spectrum Techniques in Optical Comunication
}

\author{
Bharat Vashistha ${ }^{1}$, Nishant Panwar $^{2}$, Rebala Neel Reddy ${ }^{3}$, A. Jabeena ${ }^{4}$ \\ ${ }^{1,2,3,4,}$ School of Electronics Engineering, VIT University, Vellore, Tamil
}

\begin{abstract}
Method for applying spread-spectrum techniques to optical communication is presented. The interference suppression capability of spread-spectrum systems is shown to be enhanced by optical transform domain processing.Effects of jammer in DSSS communication system are demonstrated in thispaper.Several possible implementations of this system are suggested, and applications to fiber optics, laser radar, free space optical communications, and other systems are discussed.
\end{abstract}

Keywords: additive white gaussian noise, orthogonal frequency division multiplexing, bit error rate

\section{Introduction}

SPREAD-SPECTRUM communication consists of transmitting a given signal by modulating the informationwith a large bandwidth, coded waveform such as a pn sequence. The transmitted signal occupies a bandwidth much larger than the information bandwidth. Such systems possess a number of special properties which distinguish them from narrowband communication techniques. A primary advantage of such systems is resistance to jamming and interference. A broad spectral bandwidth signal is more difficult to distinguish from ambient noise, which adds to the security of the channel.Spread-spectrum techniques have not been utilized in optical communication systems, despite their increasingpopularity and inherently wide bandwidths, due to a lackof effective modulation and coding methods available atoptical frequencies. We propose several methods for incorporating spread-spectrum techniques into optical communications. An architecture for optical spreadspectrum encoding is developed which performs pn code modulation on the Fourier transform of the data sequence. This eliminates the need for high-frequency $(\mathrm{GHz})$ optical modulators, as required by time domain pn code systems. We also present a correlation receiver design for the optical spread-spectrum system, which is simpler than the corresponding time domain receiver

Several possible implementations of these designs are suggested, as well as various applications including fiber optic communications, laser radar, optical code division multiple accessnetworks, and laser range finding.

A typical direct sequence spread-spectrum communications system is shown in Fig. 1. An information sequence, $\boldsymbol{S}(\boldsymbol{t})$, is modulated by a pn code sequence, $\boldsymbol{c}(\mathbf{f})$. The

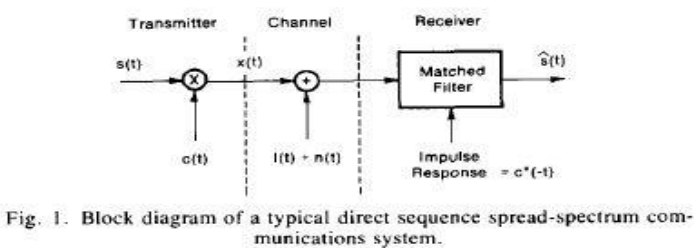

modulated signal is corrupted in the communicationschannel by interference, $I(t)$, and additive, almost whiteGaussian noise, $\boldsymbol{n}(\boldsymbol{t})$. The corrupted signal is recovered by a matched filter containing the code sequence.The ability of a spread-spectrum system to resist jammingis determined by the processing gain, which in turn is given by the ratio of transmission bandwidth to data bandwidth.

Large processing gains provide a high degree of jamming immunity. Since processing gain cannot be increased indefinitely, it is desirable to supplement the jamming resistance. This has led to the use of transform domain processing techniques. A transform domain receiver is shown in Fig. 2. The received signal is $\boldsymbol{x}(\boldsymbol{r})=\boldsymbol{S}$ $(\boldsymbol{r}) \boldsymbol{c}(\boldsymbol{r})$, plus channel interference and noise. Filtering by the transfer function $\boldsymbol{H}(w)$ is performed by multiplication followed by inverse transformation. This real-time frequency domain multiplication has been demonstrated both theoretically and experimentally. An alternate receiver implementation replaces the matched filter by multiplication with the complex conjugate of the signal spectra in the transform domain.

Transform domain-processing techniques effectivelysuppress narrow band jammers in a spreadspectrum system. The jammer may be removed by the system illustrated in Fig. 3. Input consists of the code and jammer on an RF carrier; a high power* narrowband jammer appears as an impulse in the transform domain. A gating function removes the portion of the spectrum containing the jammer. The gate output is the pn code; 
since the code has a large bandwidth, the notch filter has not seriously degraded the signal spectrum. Correlation is performed by multiplication in the transform domain, followed by an inverse Fourier transform.

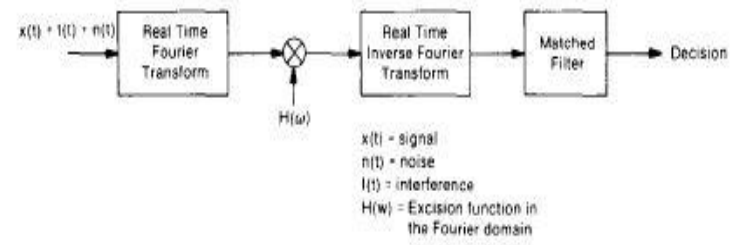

Fig. 2. General form of a transform domain receiver

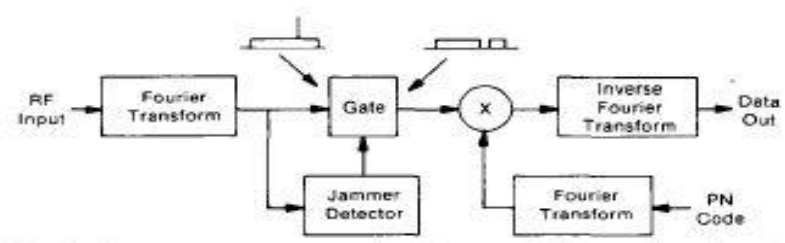

Fig. 3. System to remove narrowband jammer in the transform doman.
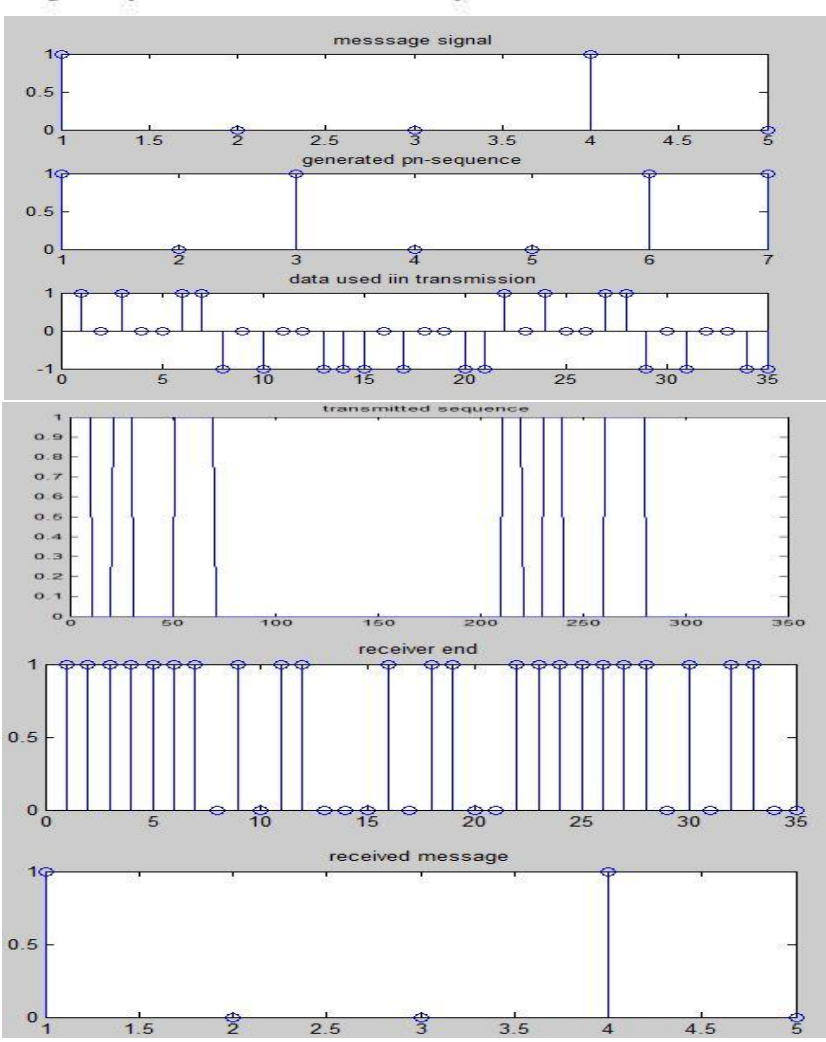

II. Transform Domain Processing In The Optical Domain

Optical signal processing techniques are suited to applications in transform domain processing. We shall consider the Chirp Transform, which can be used to implement a real-time Fourier transform. The Chirp Transform is illustrated by Fig. 4(a). An arbitrary signal $\mathrm{f}(\mathrm{t})$ is multiplied by a down-chirp, then passes through a linear system whose impulse response is an up-chirp. The result is multiplied by a down-chirp; the output is the Fourier transform of the input. This is also known as the multiply- convolve- multiply (MCM) algorithm. The system of Fig. 4(b) performs the same operation by a dual process called the convolve-multiply-convolve (CMC) algorithm. In both cases, the same result holds if the impulse responses are down-chirps and we multiply by the up-chirps. If the input is $F(w)$, the algorithms invert the Fourier transformto yield $f(-t)$.Either algorithm can be used in optics to perform spatial Fourier transforms of optical signals. The MCM algorithm may also be applied to optical pulse compression systems. This algorithm can be realized in the time domain, which forms the basis for the use of spread-spectrumsystems at optical frequencies. 

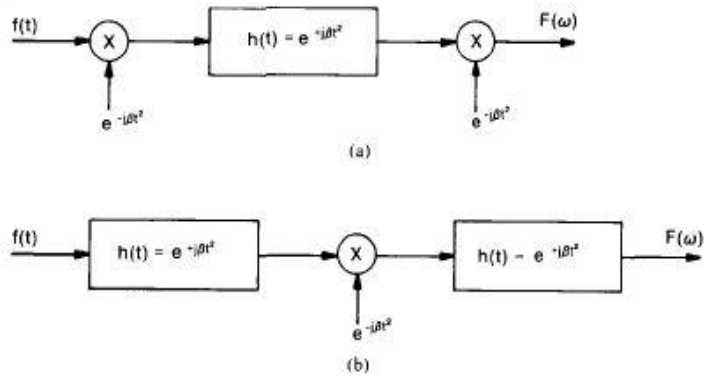

Fig. 4. Block diagram of a chirp transform system. (a) MCM algorithm,

\section{Optical Pulse Compression}

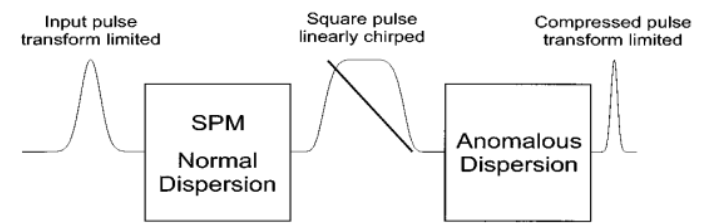

Pulse compression via fiber-grating orfiber-prism compression is first proposed by Tomlinson a fiber with positive dispersions used to broaden the pulse spectrum and generate a square intensity profile with very linear chirp across the pulse. SPM generates new frequencies and broadens the pulse spectrum, and the GVD linearizes the chirp and squares the pulse. The linear chirp can then be compensated by a dispersive element with negative dispersion (such as a grating pair, prism pair, or a chirped fiber grating operating in reflection, producing a nearly transform- limited compressed pulse. This technique was applied successfully to produce some of the shortest optical pulses. It should be stressed that this method relies on nonlinear effects and spectral broadening, which is in contrast to simple chirp compensation by devices such as chirped gratings. These chirp compensators are linear systems (similar to the second section in our compressor) that do not generate new bandwidth.

The basic configuration for optical pulse compression and shaping is shown in Fig. 5 after the treatment of [1] and [2]. Note that Fig. 5 is an optical implementation of Fig. 4(a), where $\mathrm{f}(t)$ is the pulse to be compressed. This pulse compression is achieved by inducing a chirp, or linear frequency sweep, on an optical pulse and subsequently re-phasing the chirped frequency components. In other words, the phase of the chirped signal spectrum is adjusted so that it corresponds to a narrow pulse (impulse) in the time domain. Although there are several means of obtaining chirped optical pulses, a single mode optical fiber induces uniform frequency modulation across the entire pulse profile. The frequency chirp is generated by self-phase modulation, which arises from the interaction of the propagating light and the intensity dependent portion of the fiber's refractive index .It is then necessary to re-phase the spectral components to compress the pulse in time. The system in Fig. 5 uses a diffraction grating pair as a dispersive delay time. This system can be modified to encode an optical pulse in the frequency domain. A chirped optical pulse can be produced by the nonlinear process of self-phase modulation (SPM). The fiber's refractive index is given by

$$
\mathrm{n}=\mathrm{n}_{0}+\mathrm{n}_{2} \mathrm{I}(\mathrm{t})
$$

Where $I(t)$ is the intensity profile of the light, and $n 2$ is a positive material constant [3]. The propagation constant is given by

$$
\mathrm{k}=\omega \mathrm{n}_{0} / \mathrm{c}+\mathrm{AI}(\mathrm{t})
$$

where c is the speed of light and the constant $\boldsymbol{A}$ represents a collection of terms. The phase of the optical pulse becomes

$$
\theta=\omega_{0} \mathrm{t}-\omega \mathrm{zn} \mathrm{n}_{0} / \mathrm{c}-\mathrm{AI}(\mathrm{t})
$$

where $z$ is the propagation distance. The instantaneous frequency is thus proportional to the negative time derivative of the intensity profile,

$$
\omega_{\mathrm{i}}=\mathrm{d} \theta / \mathrm{dt}=\omega_{0}-\mathrm{A} \cdot \mathrm{d}[\mathrm{I}(\mathrm{t})] / \mathrm{dt}
$$

and the properties of the resulting chirp depend on the time-varying intensity. However, for many pulse shapes of interest such as Gaussian, the time derivative of (4) leads to a non-uniform frequency chirp. Thus, the resulting frequency chirp produced by SPM alone is not linear with time over the full intensity profile. The degree of nonlinearity depends on the temporal profile of the incident optical pulse; various cases have been treated in the literature. The linearity of the chirp can be improved by the effect of positive group velocity dispersion (GVD) in the fiber. This effect is calculated by expanding 


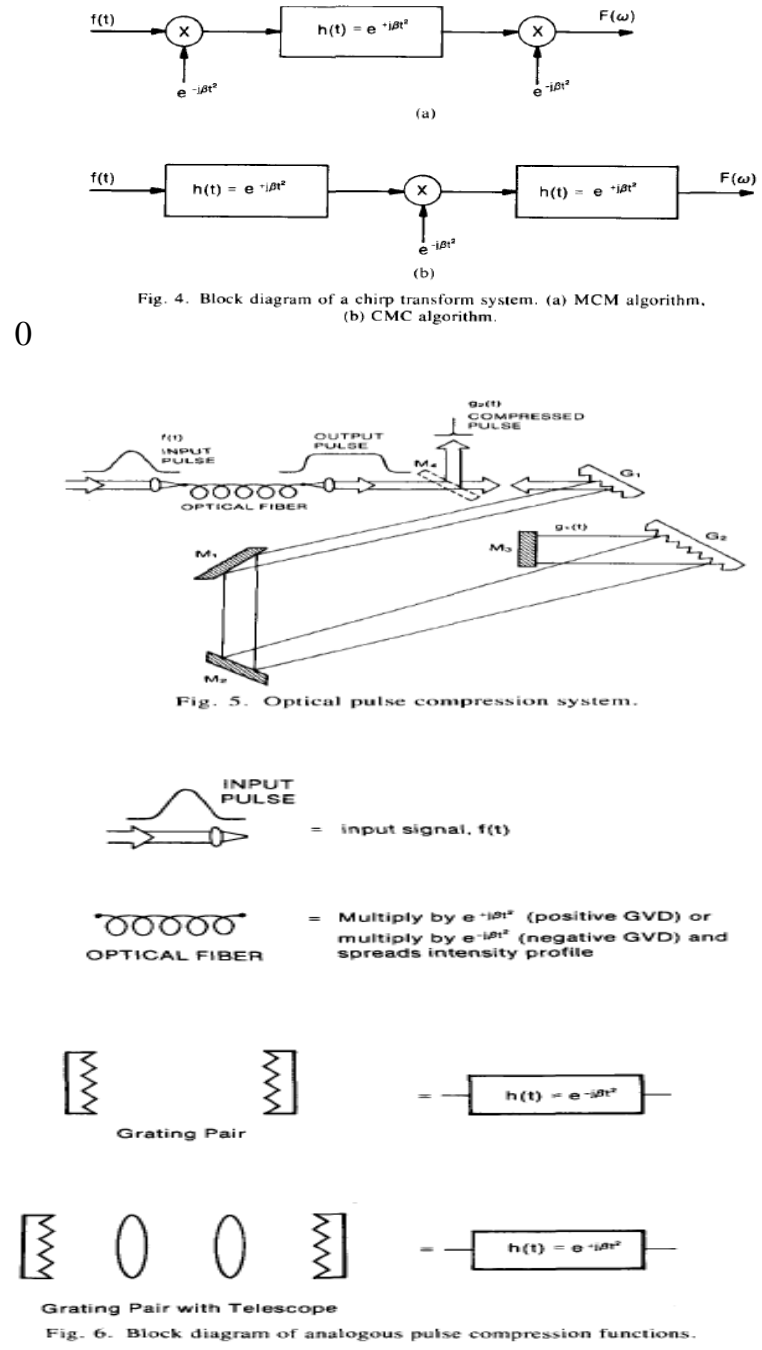

the propagation constant, $\boldsymbol{k}(\mathrm{w})$, about the center frequencywthe relevant term is

$$
\beta=\left(\partial^{2} \mathrm{k} / \partial \omega^{2}\right) \mathrm{z}=\mathrm{k}_{2} \mathrm{z}
$$

The combined effect of SPM and G V D is called disper-sive self-phase modulation (DSPM);it produces an approximate square, linearly up-chirped pulse from a single frequency input of non-uniform intensity. Once again, the required non-uniform intensity profile may be Gaussian, hyperbolic secant squared, or some other profile whose time derivative [as given in(4)] is sufficiently pro-nounced.

Asmentioned before, the system of Fig. 5 implements the following relation:

$$
\left(\mathrm{f}(\mathrm{t}) e^{-j \beta t^{2}}\right) * e^{-j \beta t^{2}}=\mathrm{F}(\omega) e^{-j \beta t^{2}}
$$

where $\boldsymbol{F}(w)$ is the Fourier transform of $\mathrm{f}(\mathrm{t}), w=2 \pi f$ is the angular frequency, and $\beta$ is a chirping factor to be determined.The chirp parameter for a grating pair is given by[14]

$$
\beta=\pi(\nabla \mathrm{f})^{2} \cdot \mathrm{d} / \mathrm{bT}
$$

where $\mathrm{d}$ is the grating constant $=\mathbf{1}$ /number of lines per mm, $b$ is the grating separation, and $T$ is a constant determined by the angle of incidence. Thus, the system of Fig. 5 realizes (6); the analogous functions for each component are shown in Fig. 6. The time-bandwidth product of a grating pair delay line may also be expressed in terms of the grating's physical properties as

$$
(\nabla \mathrm{f})(\nabla \tau)=\mathrm{mbT} / \mathrm{d} *(\nabla \mathrm{f} / \mathrm{f})^{2}
$$

where $m$ is the diffracted order. Note that the time-bandwidth product may be increased by using higher diffracted orders; this could be realized with blazed diffraction gratings, designed to diffract most of the optical power into higher orders. After one pass through the grating pair, the optical signal, $g_{1}(t)$, is given by

$$
\mathrm{g}_{1}(\mathrm{t})=\mathrm{F}(\beta \mathrm{t} / \pi) e^{-j \beta t^{2}}
$$

The first pass through the gratings has taken the Fourier transform of the original input signal, because the grating pair input had the opposite chirp as the impulse response, 
$h(t)$, of the grating pair. The signal of (6) now possesses a chirp of the same sign as $h(t)$,if this signal reflects from the mirror and transverses the grating pair a second time,no further Fourier transformation can occur. This second pass through the gratings doubles the chirp factor; the final output of the system, $\mathrm{g}_{2}(\mathrm{t})$, is

$$
\mathrm{g}_{2}(\mathrm{t})=\mathrm{F}(\beta \mathrm{t} / \pi) e^{-2 j \beta t^{2}} \quad(10)
$$

The system has performed pulse compression as an MCM system. After one pass through the gratings, the Fourier spectra of the signal are spatially separated in a plane. By placing some form of transmission mask in this

plane, it is possible to modulate the frequency components of the optical signal .If a transmission mask function $M(f)$ is placed in this plane as indicated by Fig.7,then the final output will be

$$
\mathrm{g}_{2}(\mathrm{t})=\mathrm{F}(\beta \mathrm{t} / \pi) \mathrm{M}(\beta \mathrm{t} / \pi) e^{-2 j \beta t^{2}}
$$

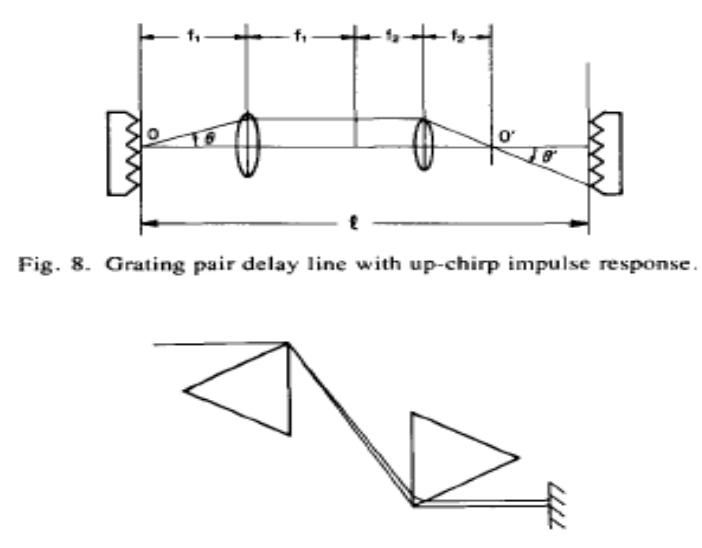

Fig. 9. Prism pair delay line with down-chirp impulse response.

\section{Spread Spectrum Techniques In Optical Communication}

There are many possible designs for an optical spread spectrum communication system based on the pulse compression architecture described previously. Fig. 7 illustrates how the chirped light may be modulated with binary data; an optical signal with positive GVD is assumed, although negative GVD may also be realized as discussed earlier. The appropriate pn code must now be impressed upon these data. One implementation involves placing a transmission-type mask between the grating pair and the feedback mirror, as shown in Fig. 7. Since the frequency components are spatially distributed in this plane, it is possible to perform both amplitude and phase modulation on the optical signal. The optical signal passes through the transmission mask twice; the second pass can only be neglected if a binary amplitude mask is used (consisting of either opaque or transparent pixels). If more complicated masks are required, then the square root of the desired amplitude function must be implemented on the mask. Any type of phase coding must account for the double phase delay incurred by a second pass through the mask. The optical signal at the encoding plane is the Fourier transform of the input pulse; thus, the encoding mask must be the Fourier transform of the desired code. The encoded optical pulse is transmitted along a fiber optic link. An optical receiver and decoding scheme for this signal is shown in Fig. 10. The optical signal is passedthrough a grating pair, which spatially separates the frequencycomponents without affecting the encodedsignal.The grating pair is now separated by twice the distance provided at the encoder, to account for the doubled chirp rate. This spatially dispersed signal is then passed through the complex conjugate of the encoding mask, $M^{*}(\mathrm{f})$. Multiplication in the transform domain is equivalent to correlation in the time domain. If the decoding mask matches the signal modulation, a correlation peak will be observed. Otherwise, the output will resemble random noise, since the cross correlation of two different codes is near zero. The optical signal passes through another grating pair to compress the spectra before detection. The correlation receiver design is greatly simplified in the transform domain. Spread-spectrum coding techniques at optical frequencies can be implemented in this way.Note that transmitting the encoded optical pulse along a fiber optic cable will not necessarily induce any further frequency chirp. The encoded optical pulse will be of lower intensity and will not exhibit a pronounced intensity variation with time, so the SPM described by (4) would not be significant. If the transmission characteristics are such that further chirping does occur, then the chirp parameter of the received signal will not match the chirp parameter of the receiver grating pair. Thus, when the received signal is spread out by the grating pair, it will not be properly aligned with the transmission mask and may not be correctly decoded. However, from (7), the chirp parameter of the receiver grating pair can be adjusted by changing the grating spacing. In this manner, the receiver can be tuned to match the chirp parameter of the received signal. This assumes that the additional chirp induced during transmission is known, or can be 
calculated from knowledge of the transmission channel properties. An alternative approach eliminates the second pass through the grating pair at both the transmitter and receiver. This second pass serves to recompress the optical spectra spatially prior to transmission, and also doubles the chirp rate. In the system of Fig. 11, a lens is placed behind thetransmission mask to spatially recombine the spectra. The transmitted signal retains its original chirp rate, so that the grating spacing at both the transmitter and receiver may be the same. The receiver design is similar to Fig. 10; a grating pair spatially disperses the opticalpulse, which passes through a decoding mask. A lens replaced the second grating pair to compress the spatially distributed pulse for optical detection. This system would require a nearly "ideal" lens at both the transmitter and receiver-a lens possessing a large field of view and practically no chromatic dispersion. Compound lenses of this type are available, although they are much more expensive than high-quality diffraction gratings.

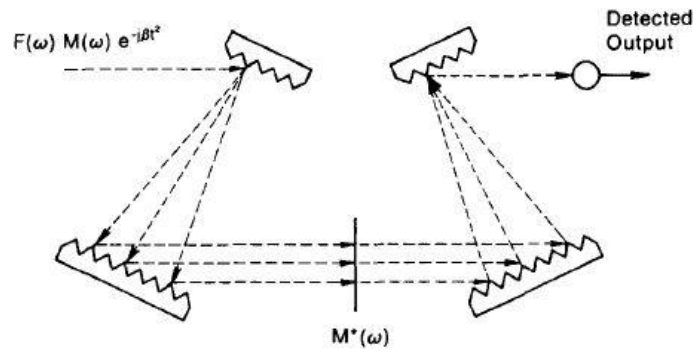

Fig. 10. Receiver for optical frequency coding system.

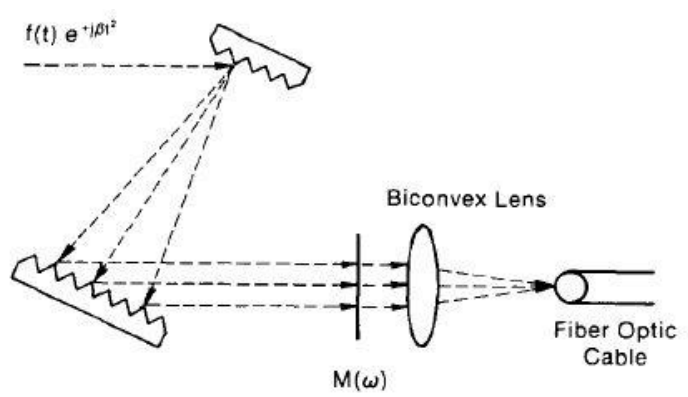

Fig. 11. Optical coding system using a lens.

In addition, the lens system should focus most of the light within the fiber's numerical aperture; this implies that fibers with high numerical apertures are desirable for this particular system realization. If the fiber does not capture all of the focused light, the resulting loss will have a radial dependence. Because the spectral components of the light now have an angular dependence, the lens and fiber combination could act as a filter, rather than a simple light collector, if they are not properly designed. If optical signals in the realm of negative GVD are used, a telescope is inserted between the transmitter grating pair as discussed earlier. This system implements the dual form of (6), namely,

$\left(\mathrm{f}(\mathrm{t}) e^{-j \beta t^{2}}\right) * e^{-j \beta t^{2}}=\mathrm{F}(\omega) e^{-j \beta t^{2}} \quad$ (12)

and the transmitter output will be of the form of (1) with an up-chirp. A transform domain receiver similar to those described earlier may be used for this signal. These systems transmit coded optical pulses in the transform domain, and decode them in the same manner. For some applications, we may transmit the temporal form of the coded pulses. For example, Bar-David and Salzhave described a balanced dual heterodyne detection scheme for phase modulated signals in the time domain; phase noise is reduced at the expense of amplitude noise, which is more easily controlled. A time domain transmitter must perform an inverse Fourier transform of the encoded signal; this may be done using two grating pairs whose impulse responses are chirps of opposite sign. A time domain transmitter is illustrated in Fig. 12, assuming an optical signal with positive GVD. After passing through the encoding mask, the signal is passed through a second grating pair whose impulse response is an up-chirp; this inverts the Fourier transform of the coded signal. The system output is given by

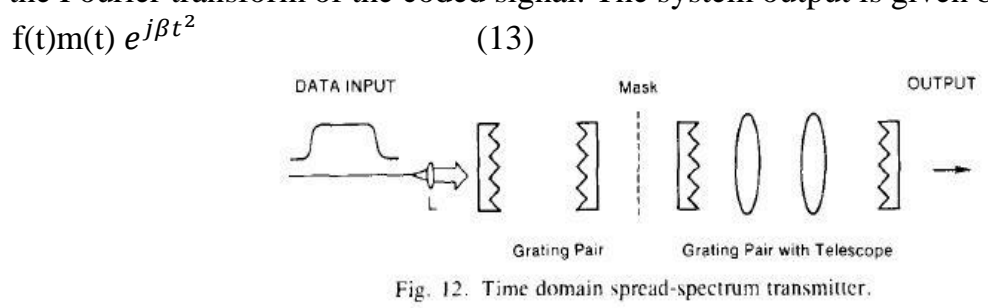

If the original optical signal is in the realm of negativeGVD, the telescope system must be placed inside the first grating pair rather than the second.The received signal is passed through a grating pair whose impulse 
response is a down-chirp; this take the Fourier transform of the received signal. An appropriate decoding mask is used as before in the transform domain, and the decoded signal may be spatially compressed using either a lens or another grating pair) for detection. A receiver for negative GVD signals uses the same design, with a telescope system between the receiver grating pair. In other words, the receiver impulse response must now become an up-chirp in order to Fourier transform the received signal. There are other possible designs for time domain transmitters and receivers which do not require the up-chirp grating pair, although they are generally more complicated. There exists another method for generating optical pulses of arbitrary shape which is different from the precedingsystems, as illustrated in Fig. 13. The pulse shape is controlled by a programmable electro-optic modulator at the system input. The optical pulse is modulated and amplified prior to passing through the spectral rephrasing process. The system shown in Fig. 13 also includes a beam splitter to sample the light after its first pass through the grating pair. The transmitted portion of the beam passes through the spatial mask, while the sampled beam is not modulated. Both beams are then recombined and directed back through the grating pair. In this manner, the un-modulated light acts as a reference pulse to sample the modulated pulse; a cross-correlation measurement may then be performed at the system output to determine the temporal shape of the modulated light. Modulating the pulse before it enters the pulse compression stage controls the resulting pulse shape and spectral content. The analysis of this system is similar to our earlier discussion; this method represents an alternative way to achieve coding of the optical pulses. The electrooptic modulator must be clocked at a rate equal to the fiber optic cable's data rate to achieve practical modulation. Since the optical data rate may approach several gigabits/ second, the processing speed would be limited by the electrooptic modulator (a few hundred megabits /second). This is a disadvantage compared to systems which real-time encoding using transform domain techniques.

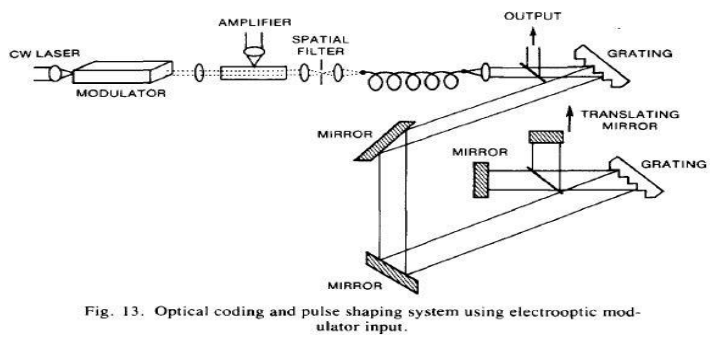

\section{Applications}

There are many applications for transform domain processing in the optical regime. One possibility is a fiberoptic communication system using spread-spectrum techniques. Fiber optics represents a secure method of communication because of its resistance to electromagnetic interference; the use of data encryption techniques can further add to the security of the channel. As another alternative, the high bandwidth of an optical fiber system may be fully exploited to provide maximum security by employing spread-spectrum optical encoding techniques. The concept of a free space laser communication system has been proposed; laser signals suffer from degradation caused by pulse spreading or scattering in the atmosphere. In order to transmit information reliably under these conditions,some form of coding is highly desirable. Although specific coding schemes for this application remain the subject of ongoing research, spread-spectrum codes are being considered because their phase coding compensates for some types of atmospheric-induced phase noise.

Another application is laser radar systems. A fundamental problem in conventional radar systems is the decrease in returning signal amplitude with range. Although the problem can be overcome by the use of larger antennas and higher transmission power levels, other researchers have noted that there are practical limits to this approach. Pulse compression techniques combined with spectral weighting have been used in microwave radar systems to overcome this problem. By transmitting a chirped pulse, it is possible to use compression techniques to concentrate the energy of the returning echo into a detectable signal. Similar techniques would be effective in laser radar systems, provided the optical pulse spectra are encoded.

A closely related problem is satellite laser range finding (SLR), which involves high-accuracy measurements of the range between a ground station and an orbiting satellite. Such measurements not only provide accurate position location, but also supply data on areas such as tectonic platemotion and variations in Earth's rotation rate. A global network of both fixed and mobile SLR ground stations is currently in use, most notably NASA's Laser Geodynamics Satellite. A technique using pseudonoise coded laser pulses to improve the accuracy of SLR has recently been proposed, and it has been shown that a maximum-likelihood receiver for estimating target range is a correlation receiver. A theoretical analysis, including noise effects for rough and smooth targets, indicates that a 1023 bit pn code yields range accuracies on the order of $1 \mathrm{~cm}$; this represents another application for our system, since improved accuracy should result from using longer pn codes. 
The coding of optical pulses also has applications in fiber optic code division multiple access systems. The scheme uses the excess bandwidth of fiber optics to map low information rate electrical or optical signals into high data rate optical pulse sequences. Single-mode fibers are well suited to this application. The sequences can be used to achieve random, asynchronous access to a communication system, free of network control among many users. Each user of a multiple-access system is provided with a unique code, whose cross correlation with other system codes is nearly zero. A set of Optical Orthogonal Codes has been developed for this purpose. A coding system which operates at optical frequencies is essential to such a system. Because optical transform domain processing has the potential to compensatefor transmission channel characteristics, as well as being highly resistant to noise and interference, it is suited to optical transmission over long distances and possesses several novel applications to outer space systems. The Strategic Defence Initiative Organization has expressed a need for the transmission of high intensity, short duration laser pulses. This problem is analogous to a longrange radar system; a similar solution involves generating lower intensity laser pulses encoded with a frequency chirp. Such pulses could be directed to a target by reflection from a pulse compression system, producing laser pulses of shorter duration and higher intensity. Optical transform domain encoding would be necessary to reduce the side lobes of the compressed pulse. Smaller scale pulse compression systems have provided compression factors of 3000 or more. Systems of this type would be effective if ground based lasers were used to supply orbital satellites equipped with large mirrors and diffraction grating compressors. The problems of high energy beam propagation through the atmosphere could be minimized by the use of optical transform domain coding.

\section{Conclusions}

We have described a system for implementing spread spectrum communication techniques at optical frequencies. The frequency encoding is achieved by a modified optical pulse compression system using transform domain processing techniques. This system is able to exploit the high bandwidth of optical communication systems; different optical implementations may be realized. Applications include improved fiber optic code division multiple access, free space optical communication systems, laser radar, integrated optical systems, and space applications such as NASA's Apollo Lightcraft project.

\section{Reference}

[1]. D. Grischkowsky and A. C. Balant, “Optical pulse compression based on enhanced frequency chirping,” Appl. Phys. Lett., vol. 41, pp.1,2-1982

[2]. A. M. Johnson, R. H. Stolen, and W. M. Simpson, " 80 x singlestate compression of frequency doubled Nd-yttrium aluminum garnet laser pulses," Appl. Phys. Letr., vol. 44, pp. 729-731, 1984.

[3]. H. A. Haus, Waves and Fields in Optoelectronics. Engelwood Cliffs, NJ: Prentice Hall, 1984.

[4]. P. Maine, D. Strickland, P. Bado, M. Pessot, and G. Mourou, "Generation of ultrahigh peak power pulses by chirped pulse amplification, ”IEEE J. Quantum Electron., vol. 24, pp. 398-403, 1988.

[5]. P. Das et al., "Surface acoustic waveacousto-electro-optic effect, "Appl. Phys. Left., vol. 49, pp. 1016-1018, 1986.

[6]. D. H. Douglas-Hamilton, A. R. Kantrowitz, and D. A. Reily, "Laseassisted propulsion research," in Radiation Energy Conversion inSpace, K. W. Billman, Ed. Washington, DC: AIAA, 1978

[7]. T. W. Bristol, "Review of spectrum analysis with SAW chirp transformsand filter bands," Case Studies in Advanced Signal Processing,pp. 226-231, Sept. 1979.

[8]. I. Bar-David and J. Saly, "On dual optical detection: Homodyne and transmitted reference heterodyne reception," IEEE Trans. Communvol. 36, pp. 1309-1315, 1988.

[9]. A. Salehi and C. A. Brackett, "Fundamental principles of fiber optics code division multiple access (F CDMA)," in Proc. IEEE Int. Conf.Commun'87, vol 3, 1987, pp 1601-1609.

[10]. J. K. Holmes. Coherent Spread spectrum systems 